\title{
Petri-net-Framework: modeling and simulation of biological networks based on Petri-nets
}

\author{
R. Hofestädt*, C. Brinkrolf \\ AG Bioinformatics and Medical Informatics, University Bielefeld, Technical Faculty, Germany \\ *e-mail: ralf.hofestaedt@uni-bielefeld.de
}

Key words: Petri-Net, simulation, new tool

Motivation and Aim: Based on a short introduction of Petri-nets and an overview of existing simulation shells this presentation will focus to a new Petri-net simulation shell based on the OpenModelica software tool. A user interface will be presented, which allows the access to the Petri net library (PNlib) ofOpenModelica. The PNlib-Shell provides a powerful simulation environment for the simulation of biological networks. Based on this new shell Petri net models can be easily created, simulated and analyzed. In addition the new system includes basic features to check and evaluate the model and to analyze simulation results generated by the simulation back-end.

\section{References}

1. Hofestädt R. (2017) Advantages of Petri-Net modeling and simulation for biological networks. J. Bioscience, Biochemstry Bioinformatics. 7(4):221-229. DOI 10.17706/ijbbb.2017.7.4.221-229. 